\title{
Circuit
}

Musiques contemporaines

\section{Document. Alberto Ginastera au Centro Latinoamericano de Altos Estudios Musicales (CLAEM). Correspondance 1961-1970 ${ }^{1}$}

\section{Laura Novoa}

Volume 17, numéro 2, 2007

Plein sud : Avant-gardes musicales en Amérique latine $\mathrm{au} \mathrm{xx}^{\mathrm{e}}$ siècle

URI : https://id.erudit.org/iderudit/016837ar

DOI : https://doi.org/10.7202/016837ar

Aller au sommaire du numéro

Éditeur(s)

Les Presses de l'Université de Montréal

ISSN

1183-1693 (imprimé)

1488-9692 (numérique)

Découvrir la revue

Citer cet article

Novoa, L. (2007). Document. Alberto Ginastera au Centro Latinoamericano de Altos Estudios Musicales (CLAEM). Correspondance 1961-1970 ${ }^{1}$. Circuit, 17(2),

35-41. https://doi.org/10.7202/016837ar d'utilisation que vous pouvez consulter en ligne. 


\title{
Alberto Ginastera au Centro Latinoamericano de Altos Estudios Musicales (CLAEM)
}

\author{
Correspondance 1961-1970
}

Laura Novoa

Ce qui suit est une sélection de lettres extraites de l'abondante correspondance inédite échangée entre le compositeur Alberto Ginastera, directeur du CLAEM, et des personnalités du monde musical international pendant les presque dix ans de labeur ininterrompu de cette institution.

Cette correspondance, conservée dans les archives du ClaEm au sein de l'Instituto Torcuato Di Tella à l'Université Torcuato Di Tella de Buenos Aires, visait principalement à promouvoir et à diffuser les activités du Centre dans le milieu musical international, ainsi qu'à attirer les compositeurs les plus importants d'Europe et des États-Unis pour qu'ils viennent prononcer des conférences ou assurer des cours en tant que professeurs invités de la chaire de composition.

Dans la plupart des lettres reviennent les références aux objectifs du Centre, les réflexions de Ginastera sur son travail et celui de ses collègues, de brèves discussions sur le contenu de la programmation des concerts et des festivals, certains problèmes institutionnels (surtout à partir de 1966), et des détails sur la gestion administrative des invitations des musiciens étrangers.

Aussi, mais dans une moindre mesure, les opinions des compositeurs invités sur leur propre musique et celle de leurs collègues. Le contenu des lettres est particulièrement éclairant pour comprendre la réalité musicale latino-américaine des années 1960, ses liens avec la musique de l'avant-garde européenne et nord-américaine, et son étroite relation avec le projet de modernisation alors en cours dans toute la région.
1. Les lettres ici présentées font partie des sources primaires d'une recherche en cours centrée sur l'histoire du Centro Latinoamericano de Altos Estudios Musicales en tant que projet de rénovation esthétique du champ musical argentin et latino-américain pendant les années 1960. L'auteure remercie Claudio Castro pour les traductions du français vers l'espagnol, et Esteban Buch pour les traductions de l'espagnol vers le français, utilisées dans le cadre de cet article. 
Pour l'édition de cette sélection, j’ai travaillé avec les originaux des archives, et j'ai choisi d'en citer les textes intégraux par ordre chronologique. La disposition des en-têtes et des formules de salutations est reproduite le plus fidèlement possible. Les passages soulignés le sont dans les documents. Les fautes d'orthographe et les coquilles dues notamment à l'absence d'accents français sur les machines à écrire argentines ont été conservées, sans faire l'objet de l'indication « $[$ sic $] »$.

Les notes, à caractère informatif, donnent des précisions sur les noms, œuvres et événements cités.

\section{Alberto Ginastera à Olivier Messiaen}

Buenos Aires, 9 août 1962

M.Olivier Messiaen

13, Villa du Danube, Paris

Cher Maitre,

J'ai un grand plaisir a vous écrire apres tant d'années que nous ne nous soyons pas vus - la derniere fois c'était a Rome en 1959 - pour vous communiquer la création d'un nouveau Centre Latinoamericain d'Hautes Etudes Musicales a Buenos Aires a l'Instituto Torcuato Di Tella de l'Argentine et avec 1'appui de la Fondation Rockefeller. Ce Centre est dédié exclusivement aux études de composition musicale. J'inclus ici le feuillet de propagande qui a été publié en espagnol parce que les étudiants, 12 boursants en total, viendront de tous les pays de l'Amérique Latine. J'ai été distingué par la nomination de directeur de ce Centre ou je dicterai aussi quelques cours de composition. Nous aurons chaque année deux ou trois professeurs extraordinaires choisis parmi les personnalités plus renommées de la musique contemporaine, qui viendront pour donner des cours spéciaux. J'ai donc l'honneur de vous inviter a donner, pour la premiere année, un cours qui aura une durée de 4 semaines (8 ou 10 séances en total) pendant les mois de mai, juin, juillet, octobre ou novembre de 1963, selon votre choix. Le cours pourrait etre dédié aux problemes du rhythme a travers votre œuvre. Le Centre vous offre pour les 4 semaines trois mille dollars (u\$s 3.000.00) en total, le passage en avion d'aller et retour en France en vous payant aussi vos dépenses d'hotel et pendant votre séjour ici². Pour le Centre ce serait un grand honneur de pouvoir compter avec votre collaboration et aussi d'avoir contribué a établir votre premier contact personnel avec l'Argentine ou tant de vos oeuvres ont eté jouées et apreciées. Personnellement j’aurai un énorme plaisir de vous avoir parmi nous pour ce bref séjour.

Si vous décidez de venir on pourrait organiser aussi quelques concerts de vos oeuvres comme par exemple, un concert d'orgue a l'église du Sant'simo Sacramento ou il y a un orgue magnifique et un concert de musique de chambre. Comme il y a ici plusieurs orchestres symphoniques nous pourrions aussi leur demander de jouer des œuvres a vous en occasion de votre visite. Quant a la langue, vous pourrez dicter vos cours directement en français, parcequ'on le comprend tres bien ici. 
Comme je prépare une grande propagande du Centre avant de réaliser le concours de bourses pour les jeunes compositeurs, je vous prie de bien vouloir me répondre d'urgence par courrier d'avion expres ou si possible, par telegramme, parce que je voudrais pouvoir annoncer les noms des professeurs invités dans nos prochaines communications.

En espérant avec impatience votre réponse je vous prie, cher Maitre et ami, de me croire votre bien dévoué

Alberto Ginastera

$\mathrm{AG} / \mathrm{js}^{3}$

Inclus : 1 dépliant

Original: lettre en français, tapée à la machine.

\section{Olivier Messiaen à Alberto Ginastera}

Paris, 21 août 1962

21 août 1962

Cher Monsieur Ginastera,

Merci infiniment et de tout cour pour votre lettre. Excusez-moi d'y répondre avec un peu de retard : rentrant en France après une tournée de concerts de mes œuvres de piano et orchestre avec Yvonne Loriod (tournée qui m’a mené au Japon, puis aux U.S.A. ou au Canada), je la trouve seulement aujourd'hui.

J'accepte avec plaisir de faire à Buenos-Aires des cours sur le Rythme (en général et dans mon œuvre) pendant 4 semaines (8 cours en tout). Je pourrais faire:

La métrique grecque, les decî-tâlas de l'Inde, l'arsis o la thésis dans le plain-chant, l'accentuation chez Mozart, mes propres recherches rythmiques, les valeurs irrationnelles chez les jeunes.

Les conditions sont parfaites. Remboursement des frais d'avion aller et retour en France, remboursement des frais de séjour, plus 3000 dollars de cachet. C'est très bien. (Je pense qu'il s'agit du dollar U.S.A. valant $5 \mathrm{NF}$ français, c'est à dire 500 anciens francs français?)

J'aimerais venir avec la pianiste Yvonne Loriod. Yvonne Loriod est sans doute une des plus extraordinaires pianistes de notre temps, elle est aussi l'interprète attitrée et préférée de mes œuvres - elle est encore ma femme (mais je vous demande de ne pas en parler, car nous sommes mariés secrètement depuis peu de temps, et nous préférons le taire pour l'instant).

Il faudrait, en ce cas, qu'on lui paye son voyage d'avion aller et retour en France, plus quelques cachets.

La présence d'Yvonne Loriod permettrait toute une série de concerts de mes œuvres : c'est pourquoi j’insiste pour sa venue.

Une malencontreuse maladie d'estomac m'empêche désormais de jouer de l’orgue en public. Renonçons à l'orgue.
3. Les initiales "js" sont celles de Josefina Schroeder, secrétaire administrative du CLAEM depuis sa création jusqu'en 1969 
4. Oiseaux exotiques fut donné en création argentine au Teatro Colón de Buenos Aires le 7 juillet 1963; le 24 juin, Olivier Messiaen et Yvonne Loriod avaient interprété Visions de l'Amen créé à Buenos Aires en 1959 - et trois numéros des Vingt Regards sur l'Enfant Jésus lors d'un concert au Museo de Arte Decorativo organisé par le Mozarteum Argentino.

5. Le chef fut Rafael Frühbeck de Burgos.

6. La visite eut finalement lieu du 18 juin au 10 juillet 1963 .
On peut jouer parmi mes œuvres :

- Réveil des oiseaux, piano et orchestre (grand orchestre normal - Yvonne Loriod au piano solo - durée : 25 minutes ).

- Oiseaux exotiques, piano et orchestre (2 clarinettes, xylophone, orchestre à vents et percussions - Yvonne Loriod au piano solo - 19 personnes en tout - durée : 15 minutes).

- Visions de l'Amen à 2 pianos (Yvonne Loriod et moi-même durée : 1 heure.)

- Peut-être la Turangalîla-Symphonie? (Très grand orchestre - comportant Ondes Martenot (avez-vous Onde et Ondiste?)- piano solo Yvonne Loriod - Petite trompette en ré, glockenspiel, vibraphone, célesta, jeu de cloches en tubes - 9 percussionnistes - etc. etc.- durée : 1 heure?-10 mouvements - l'œuvre fait tout un concert $)^{4}$.

Je ne suis pas chef d'orchestre. Il faut donc un chef pour toutes les œuvres5.

Yvonne Loriod a un immense répertoire classique et moderne. Elle peut jouer les 26 concertos piano et orchestre de Mozart par cœur, la totalité des «Iberia » d'Albeniz, la totalité des «études » de Debussy, les 4 Fantaisies de Mozart, tout Chopin et Schumann, la « $2^{\mathrm{e}}$ sonate» de Boulez, «Mana» de Jolivet, etc.etc. Elle joue aussi mes Vingt Regards (20 pièces - durée totale : 1 heure et 50 minutes) et mon Catalogue d'oiseaux (13 pièces - durée totale : 2 heures?) - soit en entier, soit en extraits.

Étant ornithologue autant que musicien et rythmicien, je ne veux pas aller en Argentina sans en connaître, et noter les chants d'oiseaux. Pouvez-vous m'indiquer des disques de chants d'oiseaux et où on peut se les procurer (afin de préparer mon voyage) et l'adresse d'un ornithologue Argentin qui me piloterait dans les endroits favorables?

Reste l'époque. Il faut concilier 3 choses : a) que ce soit la saison des cours pour vous; b) que ce soit le printemps, à cause des chants d'oiseaux; c) que cela ne gêne pas ma classe du Conservatoire de Paris. Mes cours au Conservatoire de Paris vont du $1^{\text {er }}$ octobre au $1^{\text {er }}$ juillet. Donc, mai et juin sont a rejeter, ainsi que novembre. Juillet 1963 serait bon (mais je pense que c'est l'été pour nous, et l'hiver pour vous? donc, pas d'oiseaux).

Octobre est-il le printemps pour vous? Si oui, Octobre 1963 serait le meilleur : en ce cas, je demanderai une permission spéciale a mon Directeur pour manquer les examens d'entrée et commencer ma classe au conservatoire de Paris en novembre, ce qui est possible ${ }^{6}$. Attendant votre réponse sur tous les points, je vous prie de croire, cher Monsieur Ginastera, à tous mes remerciements et à mes sentiments profondément reconnaissants et dévoués.

\section{Olivier Messiaen \\ Olivier Messaien \\ 13 Villa du Danube \\ Paris $\left(19^{\mathrm{e}}\right)$ \\ France}

Original: lettre en français, manuscrite. 


\section{Alberto Ginastera à Bruno Maderna}

Buenos Aires, 19 novembre 1963

Maitre Bruno Maderna

Adolf Spiess Strasse 5, Darmstadt, Bundesrepublik, Deutschland.

Cher Maitre,

J'ai été enchanté d'apprendre votre prochaine visite a Buenos Aires pour diriger une série de concerts de musique contemporaine au Théatre Colon. Je suis sur que vos concertes auront une grande repercussion dans notre ville et que pour vous ce voyage en Amérique Latine sera une expérience intéressante.

Comme Directeur du Centre Latinoaméricain de Hautes Etudes Musicales a l'Institut Torcuato Di Tella j'ai le plaisir de vous inviter a donner un petit cours de 5 ou 6 classes sur phonologie expérimentale avec des illustrations musicales en bandes de vos œuvres et aussi d'autres de compositeurs contemporains ${ }^{7}$. Le Centre, qui compte avec l'appui de la Fondation Rockefeller et qui a commencée a fonctionner depuis l'année 1963 , donne instruction musicale supérieure a 12 boursiers appartenant a differents pays de l'Amérique Latine qui obtiennent leurs bourses par concours. J'inclus ici un feuillet de propagande par lequel vous pourrez voir que notre centre n'est pas seulement une maison d' études, mais un vrai centre pour la recherche et la diffusion de la musique de notre temps.

Je connais tres bien vos œuvres (toutes celles qui sont enregistrées ou éditées) et justement j'ai analysé avec mes éleves votre «Serenate nº 2 » qui me parait une œuvre magnifique. Ce serait donc un grand honneur pour nous de pouvoir compter avec votre collaboration pour la saison de 1964. Je voudrai beaucoup que vous expliquiez a nos jeunes boursiers vos expériences personnelles et aussi vos idées sur les possibilités futures de notre art.

Les éleves qui sont tous des compositeurs avec une certaine expérience professionnelle ont commencé cette année en recevant des classes de : Aaron Copland, Olivier Messiaen et Riccardo Malipiero et moi-meme. Pour l' année prochaine nous avons engagé : Luigi Dallapiccola, Heinrich Ströbel ${ }^{8}$, Witold Lutoslavski ${ }^{9}$ et Gilbert Chase qui partageront avec moi (et avec vous si vous acceptez a nous rejoindre) les cours de l' année 1964 .

Quant a la question de vos versements j’ arrangerai cela avec votre empresario d'accord avec vos instructions et aussi je connait les dates de vos concerts et de vos repétitions.

J'aimerai beaucoup organiser pendant votre séjour a Buenos Aires un concert a l'Institut comme homage a vous avec vos œuvres liste possible de vos compositions pour obtenir les musiques en avance.

J'espere cher Maitre que je pourrai avoir une reponse de vous bientot et que nous aurons l' honneur et la joie de vous compter parmi nos prochains collaborateurs.

Veuillez recevoir mes salutations plus sinceres.

Bien a vous

AG/js

Original : lettre en français, tapée à la machine.
7. Maderna fut empêché d'accepter cette proposition à la suite des engagements qu'il avait pris pour la préparation de ses concerts au Teatro Colón. En revanche, il accepta de visiter l'Institut en tant qu'«invité sans caractère professionnel " et d'y faire entendre quelques-unes de ses œuvres.

8. Cette visite ne se concrétisa pas. 9. Cette visite ne se conrétisa pas non plus. 


\section{Luigi Nono à Alberto Ginastera}

Venise, 27 juin 1966

10. Nono arriva à Buenos Aires en juillet 1967 et y resta jusqu'au mois d'août.

11. Le Centre organisa deux concerts d'œuvres de musique électronique de Nono. Le 2 août 1967, furent donnés en création Omaggio a Emilio Vedova, La Fabbrica Illuminata et Ricorda cosa ti hanno fatto in Auschwitz; le 9 août, $A$ floresta é jovem e cheja de vida, avec des textes de Giovanni Pirelli et la projection d'un film documentaire sur cette œuvre réalisé au Laboratoire Électronique de la RAI de Milan et au Théâtre La Fenice de Venise. D'après la presse de l'époque, ces concerts assortis de commentaires de Nono donnèrent lieu à des débats passionnés avec le public.
Alberto Ginastera

Director, Instituto Torcuato Di Tella

Florida 946, Buenos Aires

\section{Dear Friend,}

Thank you for your letter and your telegram. By now you will have received my telegram in which I let you know that I am pleased to accept your offer to come to Buenos Aires in October ${ }^{10}$. Please excuse the long delay in answering your letter but I am still working in Milano at the electronic studio and could not make a decision earlier. I would plan to come on the $1^{\text {st }}$ of October and to stay the whole month. Please write me how and when and how many lessons there will be. How many pupils will I have? How many hours a day? How will the technical lessons be divided from the more general ones; that is: how many hours each?

I will bring several tapes. As a theme I would like to do not only "words and music" but also my recent experiences of composition in the electronic studio which is a great deal more ample than just electronic music. I would use as examples my latest three works: "Ricorda cosa ti han[no] fatto a Auschwitz" (music for "Die Ermittlung" by Peter Weiss), "La Fabbrica Illuminata" (texts by Giuliano Scabia and Cesare Pavese), and the piece I am working on now and which will be presented at the Venice Biennale Festival in September $10^{\text {thl1 }}$. Beginning with the music and words theme I will give examples from Gabrielli, Gesualdo, Bach and others up to the present time. Of my works I will start with "il canto sospeso" and continue all the way through my last experiences in the electronic studio.

I would appreciate it if you would let me know how many lessons I will be giving so that I can organize the material to fit in the given time.

I will be coming with the family, Nuria and our two daughters. There is a possibility that a friend of ours who is studying architecture in Venice will let us have apartment in Buenos Aires during our stay there. I will let you know as soon as possible.

Please dispose freely of me during my stay in your city. That is if you wish to arrange meetings or lectures or other lessons beyond what I will be doing in the institute, I will be glad to accept.

Do you have a 4 -Channel taperecorder? "La fabbrica Illuminata" is composed for a 4-Channel recorder plus a "live" mezzosoprano. If you have a mezzosoprano who could do this (even a young singer) one could do a public performance. I would help her to learn the part and in this way one could do an authentic performance. Otherwise we would have to use the tape which has the solo part incorporate in the 4-Channel tape.

If you are interested in a concert open to the public, we could do: "Ricorda cosa ti hanno fatto in Auschwitz" -11 min. (monoral tape)

"La fabbrica Illuminata" $17 \mathrm{~min}$. (4-Channel tape+mezzosoprano) new work which I'm doing for the Biennale- 40 min. 
please let me know if there is anything I can do for you and if you agree with my suggestions. With very best wishes and warm greetings to you and Mrs. Ginastera, also from Nuria,

Sincerely yours,

Luigi Nono

Dictated to me just before he left for Milano

Nuria Nono

Original: lettre en anglais, tapée à la machine. Signée par Luigi Nono et Nuria Schoenberg-Nono.

\section{Karlheinz Stockhausen à Alberto Ginastera}

Kürten, 7 décembre 1967
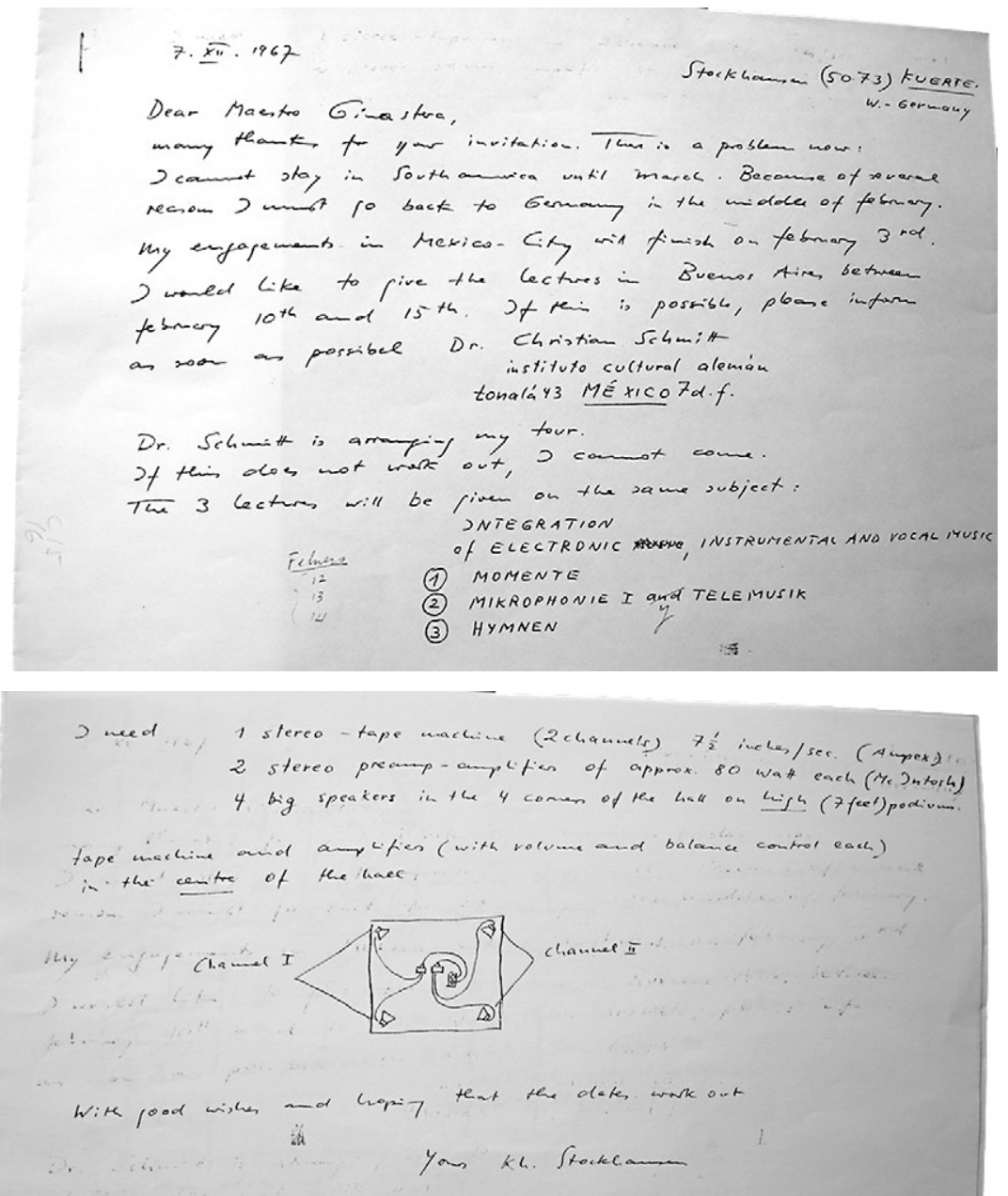

Lettre manuscrite en anglais ${ }^{12}$.
12. Stockhausen annula sa tournée en Amérique du Sud peu de temps avant la date prévue en évoquant des problèmes de santé. 